\title{
Novel Einstein-scalar-Gauss-Bonnet wormholes without exotic matter
}

\author{
Georgios Antoniou* \\ School of Physics and Astronomy, University of Minnesota, Minneapolis, Minnesota 55455, USA \\ Athanasios Bakopoulos ${ }^{\dagger}$ and Panagiota Kanti $\odot^{\ddagger}$ \\ Division of Theoretical Physics, Department of Physics, University of Ioannina, GR-45110 Ioannina, Greece \\ Burkhard Kleihaus $^{\S}$ and Jutta Kunz ${ }^{\|}$ \\ Institut für Physik, Universität Oldenburg, D-26111 Oldenburg, Germany
}

(Received 3 May 2019; published 14 January 2020)

\begin{abstract}
Novel wormholes are obtained in Einstein-scalar-Gauss-Bonnet theory for several coupling functions. The wormholes may feature a single- or a double-throat geometry and do not demand any exotic matter. The scalar field may asymptotically vanish or be finite, and it may possess radial excitations. The domain of existence is fully mapped out for various forms of the coupling function.
\end{abstract}

DOI: 10.1103/PhysRevD.101.024033

\section{INTRODUCTION}

In the quest for the fundamental theory of gravity, Einstein-scalar-Gauss-Bonnet (EsGB) theories represent interesting alternative theories of gravity (see, e.g., [1,2]). They belong to the class of quadratic gravitational theories that contain higher-curvature gravitational terms. These terms are treated as small deformations that nevertheless complete Einstein's general relativity and may modify its predictions at regimes of strong gravity. In the EsGB theory, the Einstein-Hilbert action is supplemented by a scalar field, nonminimally coupled to the quadratic Gauss-Bonnet (GB) term. The resulting field equations are of second order, avoiding Ostrogradski instability and ghosts [3-5]. In addition, this quadratic theory has so far survived the constraints set by the detection of gravitational waves emitted during the binary mergers, when the coupling function allows to set the scalar field to zero in the cosmological context, and thus lead to the same solutions as the standard cosmological $\Lambda \mathrm{CDM}$ model [6]. The study of the types of solutions that this theory admits is therefore of paramount importance.

Motivated by string theory with the dilaton as the scalar field, the Einstein-dilaton-Gauss-Bonnet (EdGB) theory

\footnotetext{
*anton296@umn.edu

†abakop@cc.uoi.gr

"pkanti@cc.uoi.gr

\$b.kleihaus@uni-oldenburg.de

"jutta.kunz@uni-oldenburg.de
}

Published by the American Physical Society under the terms of the Creative Commons Attribution 4.0 International license. Further distribution of this work must maintain attribution to the author(s) and the published article's title, journal citation, and DOI. Funded by SCOAP ${ }^{3}$. features an exponential coupling between the scalar field and the GB term [7-9]. Black-hole solutions arising in the context of the EdGB theory differ from the Schwarzschild or Kerr black holes since they possess a nontrivial dilaton field and thus carry dilaton hair [10-21]. The extended family of EsGB theories, where different coupling functions of the scalar field to the GB term may be employed, has attracted recently considerable attention [22-37]. In these theories, new black holes arise through spontaneous or induced scalarization depending on whether the scalar field acquires a zero or nonzero, respectively, value at infinity. The stability of scalarized black holes of EsGB theories has been addressed in detail by analyzing their radial perturbations and revealing a distinct dependence on the coupling function [38].

A particularly interesting property emerging in the EdGB solutions is the presence of regions with negative effective energy density - this is due to the presence of the higher-curvature GB term and is therefore of purely gravitational nature [10,39]. Consequently, the EdGB theory allows for Lorentzian, traversable wormhole solutions without the need for exotic matter [39,40]. It is tempting to conjecture that the more general EsGB theories should also allow for traversable wormhole solutions. Indeed, traversable wormholes require violation of the energy conditions [41,42]. But whereas in general relativity this violation is typically achieved by a phantom field [43-48], in EdGB theories it is the effective stress-energy tensor that allows for this violation $[39,40]$.

Thus, in the context of this work, we consider a general class of EsGB theories with an arbitrary coupling function for the scalar field. We first readdress the case of the exponential coupling function and show that the EdGB 
theory is even richer than previously thought, since it features also wormhole solutions with a double throat and an equator in between. Then, we consider alternative forms of the scalar coupling function and demonstrate that the EsGB theories always allow for traversable wormhole solutions, featuring both single and double throats. The scalar field may vanish or be finite at infinity, and it may have nodes. We also map the domain of existence (DOE) of these wormholes in various exemplifications, evaluate their global charges and throat areas, and demonstrate that the throat remains open without the need for any exotic matter.

In Sec. II, we briefly recall EsGB theory and discuss the throat geometry for single- and double-throat configurations. We here present the asymptotic expansions near the throat/equator and in the two asymptotically flat regions, and we also derive the formulae necessary to study the violation of the energy conditions. We present our numerical solutions in Sec. III and discuss some of their properties, their domains of existence, and the energy conditions. In order to impose symmetry of the solutions under reflection of the spatial radial coordinate, $\eta \rightarrow-\eta$, we study in Sec. IV the junction conditions at the throat/equator and show that we can solve these by including a shell of ordinary matter only. In Sec. V, we present embedding diagrams of the wormhole solutions, and we conclude in Sec. VI. For completeness, we show the lengthy set of field equations in the Appendix.

\section{THE EINSTEIN-SCALAR-GAUSS-BONNET THEORY}

We consider the following effective action describing a quadratic scalar-tensor theory:

$S=\frac{1}{16 \pi} \int d^{4} x \sqrt{-g}\left[R-\frac{1}{2} \partial_{\mu} \phi \partial^{\mu} \phi+F(\phi) R_{\mathrm{GB}}^{2}\right]$.

The theory contains the Ricci scalar $R$, a scalar field $\phi$, and the quadratic gravitational Gauss-Bonnet term defined as

$$
R_{\mathrm{GB}}^{2}=R_{\mu \nu \rho \sigma} R^{\mu \nu \rho \sigma}-4 R_{\mu \nu} R^{\mu \nu}+R^{2}
$$

in terms of the Riemann tensor $R_{\mu \nu \rho \sigma}$, the Ricci tensor $R_{\mu \nu}$, and the Ricci scalar $R$. The GB term, a topological invariant in four dimensions, is coupled to the scalar field through a coupling function $F(\phi)$. The form of the latter will be left arbitrary; therefore, our analysis will apply to a whole class of Einstein-scalar-GB theories described by the action (1).

The Einstein and scalar-field equations are obtained by variation of the action with respect to the metric, respectively, the scalar field, and have the form

$$
G_{\mu \nu}=T_{\mu \nu}, \quad \nabla^{2} \phi+\dot{F}(\phi) R_{\mathrm{GB}}^{2}=0,
$$

where the stress-energy tensor of the theory is given by the following expression:

$$
\begin{aligned}
T_{\mu \nu}= & -\frac{1}{4} g_{\mu \nu} \partial_{\rho} \phi \partial^{\rho} \phi+\frac{1}{2} \partial_{\mu} \phi \partial_{\nu} \phi \\
& -\frac{1}{2}\left(g_{\rho \mu} g_{\lambda \nu}+g_{\lambda \mu} g_{\rho \nu}\right) \eta^{\kappa \lambda \alpha \beta} \tilde{R}_{\alpha \beta}^{\rho \gamma} \nabla_{\gamma} \partial_{\kappa} F(\phi) .
\end{aligned}
$$

Note that $T_{\mu \nu}$ receives contributions from the kinetic term of the scalar field but also from the GB term, with the latter being nontrivial for a nonconstant coupling function $F(\phi)$ as expected. The dot above $F(\phi)$ in the scalar-field equation denotes the derivative with respect to the scalar field, and we have used the definitions $\tilde{R}_{\alpha \beta}^{\rho \gamma}=\eta^{\rho \gamma \sigma \tau} R_{\sigma \tau \alpha \beta}$ and $\eta^{\rho \gamma \sigma \tau}=\epsilon^{\rho \gamma \sigma \tau} / \sqrt{-g}$.

In this work, we consider only static, spherically symmetric solutions of the field equations. To this end, we employ the following line element:

$$
\begin{aligned}
d s^{2}= & -e^{f_{0}(\eta)} d t^{2} \\
& +e^{f_{1}(\eta)}\left\{d \eta^{2}+\left(\eta^{2}+\eta_{0}^{2}\right)\left(d \theta^{2}+\sin ^{2} \theta d \varphi^{2}\right)\right\} .
\end{aligned}
$$

The substitution of the above metric in the Einstein and scalar-field equations, given in Eq. (3), leads to three second-order and one first-order ordinary differential equations (ODEs) that are displayed in the Appendix. Due to the Bianchi identity, only three of these four equations are independent. In our analysis, we choose to solve the three second-order equations while the first-order one will serve as a constraint on the unknown quantities $f_{0}, f_{1}$, and $\phi$.

\section{A. Single- and double-throat geometry}

In our previous analyses $[39,40]$, we determined traversable wormhole solutions by employing the following line element:

$d s^{2}=-e^{f_{0}(l)} d t^{2}+p(l) d l^{2}+\left(l^{2}+r_{0}^{2}\right)\left(d \theta^{2}+\sin ^{2} \theta d \varphi^{2}\right)$.

The wormhole geometry is characterized by the circumferential radius $R_{c}$ defined as

$$
R_{c}=\left.\frac{1}{2 \pi} \int_{0}^{2 \pi} \sqrt{g_{\varphi \varphi}}\right|_{\theta=\pi / 2} d \varphi .
$$

A minimum of $R_{c}$ corresponds to a wormhole throat, whereas a local maximum corresponds to an equator. For the line element (6), the circumferential radius is $R_{c}(l)=\sqrt{l^{2}+r_{0}^{2}}$. This expression clearly possesses a minimum at $l=0$, corresponding to a throat of radius $r_{0}$, and it does not allow for a local maximum, i.e., an equator. Consequently, wormholes with only a throat and no equator were presented in $[39,40]$.

For the set of coordinates defined in Eq. (5), the circumferential radius is expressed as $R_{c}(\eta)=e^{f_{1} / 2} \sqrt{\eta^{2}+\eta_{0}^{2}}$. As we will see, this expression allows for the existence of one or two local minima (i.e., throats) and of a local maximum 
(i.e., equator). We introduce the distance variable in a coordinate-independent way as

$$
\xi=\int_{0}^{\eta} \sqrt{g_{\eta \eta}} d \tilde{\eta}=\int_{0}^{\eta} e^{f_{1}(\tilde{\eta}) / 2} d \tilde{\eta}
$$

The conditions for a throat, respectively, equator, at $\eta=0$ then read

$$
\left.\frac{d R_{c}}{d \xi}\right|_{\eta=0}=0,\left.\quad \frac{d^{2} R_{c}}{d \xi^{2}}\right|_{\eta=0} \gtrless 0,
$$

where the greater sign $(>)$ refers to a throat and the smaller sign $(<)$ to an equator. Using the metric (5), these conditions yield

$$
f_{1}^{\prime}(0)=0, \quad \eta_{0}^{2} f_{1}^{\prime \prime}(0)+2 \gtrless 0,
$$

where the prime denotes derivative with respect to $\eta$. In the degenerate case, when the throat and equator coincide, the inequalities in Eqs. (9) and (10) become equalities. If a throat/equator is located at $\eta=0$, then its area is given by $A_{t, e}=4 \pi R_{c}^{2}(0)=4 \pi \eta_{0}^{2} e^{f_{1}(0)}$, while for a double-throat wormhole with the throat located at $\eta_{\mathrm{t}}, A_{t}=4 \pi R_{c}^{2}\left(\eta_{\mathrm{t}}\right)=$ $4 \pi\left(\eta_{\mathrm{t}}^{2}+\eta_{0}^{2}\right) e^{f_{1}\left(\eta_{\mathrm{t}}\right)}$.

\section{B. Asymptotic expansions}

\section{Expansion near the throat/equator}

A traversable wormhole solution is characterized by the absence of horizons or singularities. In order to ensure that this is the case for our solutions, we consider the following regular expansions for the metric functions and scalar field, near the throat/equator at $\eta=0$ :

$$
\begin{aligned}
e^{f_{0}} & =a_{0}\left(1+a_{1} \eta+a_{2} \eta^{2}+\cdots\right), \\
e^{f_{1}} & =b_{0}\left(1+b_{1} \eta+b_{2} \eta^{2}+\cdots\right), \\
\phi & =\phi_{0}+\phi_{1} \eta+\phi_{2} \eta^{2}+\cdots .
\end{aligned}
$$

The Lorentzian signature of spacetime demands that both parameters $a_{0}$ and $b_{0}$ must be positive; in addition, they should be finite and nonvanishing. As discussed in the previous subsection, the emergence of an extremum in the circumferential radius $R_{c}$ dictates that $f_{1}^{\prime}(0)=0$; this leads to the result $b_{1}=0$. The $(\eta \eta)$ component of the Einstein equations, given in Eq. (A2), yields near the throat/equator a constraint equation,

$$
\left[\left(\eta_{0}^{2} \phi^{2}+4\right) e^{f_{1}}-8 f_{0}^{\prime} \phi^{\prime} \dot{F}\right]_{\eta=0}=0 .
$$

The remaining three equations may then be solved to express the second-order coefficients $\left(a_{2}, b_{2}, \phi_{2}\right)$ in terms of the zero and first-order coefficients in the $\eta$ expansions (11)-(13). These are found to have the form

$$
\begin{gathered}
a_{2}=\frac{b_{0}\left[4 b_{0} \dot{F}_{0}^{2} \phi_{1}^{2}\left(\eta_{0}^{2} \phi_{1}^{2}+4\right)^{2}+b_{0}^{3} \eta_{0}^{2}\left(\eta_{0}^{2} \phi_{1}^{2}+4\right)^{2}-128 \dot{F}_{0}^{2} \eta_{0}^{2} \phi_{1}^{6} \ddot{F}_{0}\right]}{256 \dot{F}_{0}^{2} \phi_{1}^{2}\left(b_{0}^{2} \eta_{0}^{2}+4 \dot{F}_{0}^{2} \phi_{1}^{2}\right)} \\
b_{2}=-\frac{2 b_{0} \phi_{1}^{2} \ddot{F}_{0}}{b_{0}^{2} \eta_{0}^{2}+4 \dot{F}_{0}^{2} \phi_{1}^{2}} \\
\phi_{2}=-\frac{4 b_{0} \dot{F}_{0}^{2} \phi_{1}^{2}\left(\eta_{0}^{2} \phi_{1}^{2}+4\right)+b_{0}^{3} \eta_{0}^{2}\left(\eta_{0}^{2} \phi_{1}^{2}+4\right)+64 \dot{F}_{0}^{2} \phi_{1}^{4} \ddot{F}_{0}}{32\left(b_{0}^{2} \dot{F}_{0} \eta_{0}^{2}+4 \dot{F}_{0}^{3} \phi_{1}^{2}\right)}
\end{gathered}
$$

From the above expressions, it seems that there are six free parameters in our theory: the coefficients $\left(\eta_{0}, \phi_{0}, \phi_{1}\right.$, $\left.a_{0}, b_{0}\right)$ and the coupling constant $\alpha$ which is defined through the relation $F(\phi)=\alpha \tilde{F}(\phi)$, where $\tilde{F}$ is a dimensionless quantity. However, the actual number of free parameters is much smaller. First of all, we notice that the field equations (A1)-(A4) are invariant under the simultaneous scaling of the coordinate $\eta$, the constant $\eta_{0}$, and the scalar-field coupling constant $\alpha$,

$$
\eta \rightarrow \lambda \eta, \quad \eta_{0} \rightarrow \lambda \eta_{0}, \quad \alpha \rightarrow \lambda^{2} \alpha,
$$

where $\lambda$ is an arbitrary constant. Therefore, we may fix $\eta_{0}$, which determines the scale of the wormhole's equator/ throat, to a specific value, or equivalently introduce a dimensionless coupling parameter $\alpha / \eta_{0}^{2}$. We can also fix three of the remaining four parameters by applying appropriate boundary conditions at infinity. Thus, by demanding asymptotic flatness, expressed by the conditions

$$
\lim _{\eta \rightarrow \infty}\left|g_{t t}\right|=1, \quad \lim _{\eta \rightarrow \infty} g_{\eta \eta}=1,
$$

we may fix the $a_{0}$ and $b_{0}$ parameters, while the condition $\lim _{\eta \rightarrow \infty} \phi=\phi_{\infty}$ allows us to fix $\phi_{1}$. Concluding, the only 
free parameters in the near-equator/throat area are the dimensionless coupling constant $\alpha / \eta_{0}^{2}$ and the value $\phi_{0}$ of the scalar field at $\eta=0$.

Let us also examine the components of the stress-energymomentum tensor near the throat/equator. We find

$$
\begin{gathered}
T^{t}{ }_{t}=\frac{1}{b_{0} \eta_{0}^{2}}-\frac{4 \phi_{1}^{2} \ddot{F}_{0}}{b_{0}^{2} \eta_{0}^{2}+4 \dot{F}_{0}^{2} \phi_{1}^{2}}+O(\eta), \\
T^{\eta}{ }_{\eta}=-\frac{1}{b_{0} \eta_{0}^{2}}+O(\eta), \\
T^{\theta}{ }_{\theta}=\frac{-b_{0} \eta_{0}^{2} \phi_{1}^{2} \ddot{F}_{0}\left(\eta_{0}^{2} \phi_{1}^{2}+4\right)+2 b_{0}^{2} \eta_{0}^{2}+8 \dot{F}_{0}^{2} \phi_{1}^{2}}{8 b_{0} \dot{F}_{0}^{2} \eta_{0}^{2} \phi_{1}^{2}+2 b_{0}^{3} \eta_{0}^{4}}+O(\eta) .
\end{gathered}
$$

We observe that, as desired, all components of the stressenergy tensor are finite at $\eta=0$, i.e., at the location of the throat or equator of the solution.

\section{Expansion at large distances}

At large values of the radial coordinate, the metric functions and scalar field are expanded in a power series form in $1 / \eta$,

$$
\begin{aligned}
& e^{f_{0}}=1+\sum_{n=1}^{\infty} \frac{p_{n}}{\eta^{n}}, \\
& e^{f_{1}}=1+\sum_{n=1}^{\infty} \frac{q_{n}}{\eta^{n}}, \\
& \phi=\phi_{\infty}+\sum_{n=1}^{\infty} \frac{d_{n}}{\eta^{n}} .
\end{aligned}
$$

In the above expressions, we have already imposed the conditions for asymptotic flatness and constant value of the scalar field. Substituting the above expansions into the field equations (A1)-(A4), we may determine the unknown coefficients $\left(p_{n}, q_{n}, d_{n}\right)$ in terms of only two coefficients that remain arbitrary: $d_{1}=-D$, where $D$ is the scalar charge of the wormhole, and $p_{1}=-2 M$, where $M$ is the Arnowitt-Deser-Misner mass of the wormhole. Thus, the number of free parameters at infinity is also two, similarly to the near-throat/equator regime. We have calculated the remaining coefficients up to order $\mathcal{O}\left(1 / r^{5}\right)$, and the asymptotic solutions have the following form:

$$
\begin{gathered}
e^{f_{0}}=1-\frac{2 M}{\eta}+\frac{2 M^{2}}{\eta^{2}}-\frac{M\left(D^{2}+36 M^{2}-12 \eta_{0}^{2}\right)}{24 \eta^{3}}+\frac{D^{2} M^{2}+12\left(M^{4}-M^{2} \eta_{0}^{2}-4 D M \dot{F}_{\infty}\right)}{12 \eta^{4}}+\mathcal{O}\left(\frac{1}{\eta^{5}}\right), \\
e^{f_{1}}=1+\frac{2 M}{\eta}+\frac{12 M^{2}-D^{2}-4 \eta_{0}^{2}}{8 \eta^{2}}+\frac{M\left[12\left(M^{2}-3 \eta_{0}^{2}\right)-5 D^{2}\right]}{24 \eta^{3}} \\
+\frac{3 D^{4}+D^{2}\left(96 \eta_{0}^{2}-104 M^{2}\right)+48\left(M^{4}-24 M^{2} \eta_{0}^{2}+7 \eta_{0}^{4}\right)+1536 D M \dot{F}_{\infty}}{768 \eta^{4}}+\mathcal{O}\left(\frac{1}{\eta^{5}}\right), \\
\phi=\phi_{\infty}-\frac{D}{\eta}-\frac{D^{3}+4 D\left(M^{2}-3 \eta_{0}^{2}\right)}{48 \eta^{3}}-\frac{4 M^{2} \dot{F}_{\infty}}{\eta^{4}}+\mathcal{O}\left(\frac{1}{\eta^{5}}\right) .
\end{gathered}
$$

We observe that the above solutions have exactly the same form as the corresponding solutions which describe asymptotically flat black holes [24]. Apparently, the emergence of an asymptotically flat limit does not depend on the choice of the boundary condition at the other asymptotic regime, i.e., the horizon of a black hole or the throat/equator of a wormhole. The main difference is that, in the case of black holes, the mass $M$ and the scalar charge $D$ are related parameters-which makes black holes a one-parameter family of solutions - while, in the case of wormholes, these two parameters are independent. Also, the aforementioned asymptotic solutions at infinity are almost independent of the functional form of the coupling function $F(\phi)$, since the latter does not enter in the expansions earlier than in the fourth order.
Finally, if we make use of the expansions above, we may calculate again the stress-energy tensor components at large distances. These are found to be

$T^{t}{ }_{t}=-T^{\eta}{ }_{\eta}=T^{\theta}{ }_{\theta}=T_{\varphi}^{\varphi} \approx-\phi^{2} / 4 \approx-D^{2} / 4 \eta^{4}+\mathcal{O}\left(1 / \eta^{5}\right)$.

As we expect, the above expressions have exactly the same form as the corresponding ones for the asymptotically flat black holes. We observe that, at large distances where the curvature of spacetime is small, the stress-energy tensor is dominated by the kinetic term of the scalar field which is itself decaying fast. 


\section{Violation of energy conditions}

In our previous works $[39,40]$, we have shown that for any single-throat wormhole the null energy condition is violated at least in some region near the throat. Here, we review that analysis and show that the violation of the null energy condition also holds for double-throat wormholes.

The null energy condition (NEC) is expressed as $T_{\mu \nu} n^{\mu} n^{\nu} \geq 0$, where $n^{\mu}$ is any null vector satisfying the condition $n^{\mu} n_{\mu}=0$. We may define the null vector as $n^{\mu}=$ $\left(1, \sqrt{-g_{t t} / g_{\eta \eta}}, 0,0\right)$ with its contravariant form being $n_{\mu}=\left(g_{t t}, \sqrt{-g_{t t} g_{\eta \eta}}, 0,0\right)$. For a spherically symmetric spacetime, the NEC takes the form

$$
T_{\mu \nu} n^{\mu} n^{\nu}=T_{t}^{t} n^{t} n_{t}+T_{\eta}^{\eta} n^{\eta} n_{\eta}=-g_{t t}\left(-T_{t}^{t}+T_{\eta}^{\eta}\right) .
$$

Then, the NEC holds if $-T_{t}^{t}+T_{\eta}^{\eta} \geq 0$. Alternatively, we may choose $n^{\mu}=\left(1,0, \sqrt{-g_{t t} / g_{\theta \theta}}, 0\right)$, and a similar analysis leads to the condition $-T_{t}^{t}+T_{\theta}^{\theta} \geq 0$.

For a wormhole solution to emerge, it is essential that these two conditions are violated [41]. Indeed, using the expansion of the wormhole solution at the throat/equator, we find

$$
\left[-T_{t}^{t}+T_{\eta}^{\eta}\right]_{\eta_{t, e}}=-2\left[e^{-f_{1}} R_{c}^{\prime \prime} / R_{c}\right]_{\eta_{t, e}}
$$

Consequently, the NEC is always violated at the throat(s), since $R_{c}$ possesses a minimum there, implying $R_{c}^{\prime \prime}\left(\eta_{t}\right)>0$, while no violation occurs at the equator, where $R_{c}^{\prime \prime}\left(\eta_{e}\right)<0$. For example, for a single-throat solution with the throat at $\eta=0$, we obtain the explicit expressions

$$
\begin{gathered}
{\left[-T_{t}^{t}+T_{\eta}^{\eta}\right]_{\eta=0}=\left[-\frac{2 e^{-f_{1}}}{\eta_{0}^{2}}+\frac{4 \ddot{F} \phi^{\prime 2}}{e^{2 f_{1}} \eta_{0}^{2}+4 \dot{F}^{2} \phi^{\prime 2}}\right]_{\eta=0}} \\
=-\frac{2}{b_{0} \eta_{0}^{2}}+\frac{4 \phi_{1}^{2} \ddot{F}_{0}}{b_{0}^{2} \eta_{0}^{2}+4 \dot{F}_{0}^{2} \phi_{1}^{2}}, \\
{\left[-T_{t}^{t}+T_{\theta}^{\theta}\right]_{\eta=0}=\left[\frac{\ddot{F} \phi^{\prime 2}\left(4-\eta_{0}^{2} \phi^{\prime 2}\right)}{2\left(e^{2 f_{1}} \eta_{0}^{2}+4 \dot{F}^{2} \phi^{\prime 2}\right)}\right]_{\eta=0}} \\
=\frac{\phi_{1}^{2} \ddot{F}_{0}\left(4-\eta_{0}^{2} \phi_{1}^{2}\right)}{2 b_{0}^{2} \eta_{0}^{2}+8 \dot{F}_{0}^{2} \phi_{1}^{2}}
\end{gathered}
$$

where we have used the approximate expressions Eqs. (20)-(22) near the wormhole throat. We note that the desired violation of the NEC follows not from the presence of an exotic form of matter but from the synergy between the scalar field and the quadratic GB term.

In the far-asymptotic regime, we may use the expansions at infinity Eqs. (26)-(28) to find that the two null energy conditions take the form

$$
-T^{t}{ }_{t}+T_{\eta}^{\eta}=\frac{D^{2}}{2 \eta^{4}}+\mathcal{O}\left(\frac{1}{\eta^{5}}\right)
$$

$$
-T^{t}{ }_{t}+T^{\theta}{ }_{\theta}=-\frac{40 D M \dot{F}_{\infty}}{\eta^{6}}+\mathcal{O}\left(\frac{1}{\eta^{7}}\right)
$$

We observe that if $D \dot{F}_{\infty}>0$, the second null energy condition is also violated at spatial infinity.

Let us also examine the weak energy condition (WEC), which suggests that the energy density measured by any observer has to be greater than or equal to zero. This is expressed through the inequality $T_{\mu \nu} V^{\mu} V^{\nu} \geq 0$, where $V^{\mu}$ is any timelike vector. If we choose $V^{\mu}=\left(1 / \sqrt{-g_{t t}}, 0,0,0\right)$, and impose the condition $V_{\mu} V^{\mu}=-1$, then $V_{\mu}=$ $\left(-\sqrt{-g_{t t}}, 0,0,0\right)$, and the WEC is simply $T_{t}^{t} \leq 0$. Near the throat/equator, we found that $T_{t}^{t}$ is given by Eq. (20); this expression is not sign definite; therefore, the WEC may also be violated in the small $\eta$ regime. On the other hand, at asymptotic infinity, where $T_{\mu \nu}$ is dominated by the kinetic term of the scalar field, the $T_{t}^{t}$ component is given by Eq. (29) and clearly obeys the WEC.

\section{NUMERICAL SOLUTIONS}

We now turn to the derivation of the wormhole solutions by numerically integrating the three second-order, ordinary differential equations (A1), (A3), and (A4). In order to find asymptotically flat, regular wormhole solutions, we have to impose appropriate boundary conditions at asymptotic infinity and at the throat/equator, as discussed in the previous section. For completeness, we list here the full set of these boundary conditions,

$$
\begin{array}{r}
f_{0}(\infty)=f_{1}(\infty)=0, \quad \phi(\infty)=\phi_{\infty}, \\
f_{1}^{\prime}(0)=0, \quad\left[\left(\eta_{0}^{2} \phi^{\prime 2}+4\right) e^{f_{1}}-8 f_{0}^{\prime} \phi^{\prime} \dot{F}\right]_{\eta=0}=0 .
\end{array}
$$

For the numerical integration, we use the compactified coordinate $x=\eta /\left(\eta+\eta_{0}\right)$ to cover the range $0 \leq \eta<\infty$. We choose $\eta_{0}=1$ for all our numerical solutions. The software package COLSYS is then used to solve the three, second-order ODEs with the aforementioned boundary conditions.

In our analysis, we have found wormhole solutions with either vanishing or nonvanishing asymptotic values of the scalar field, namely for $\phi_{\infty}=0$ and $\phi_{\infty}=1$. We have also considered several forms of $F(\phi)$, including exponential $F=\alpha e^{-\gamma \phi}, F=\alpha e^{-\gamma \phi^{2}}$, power-law $F=\alpha \phi^{n}$ with $n \neq 0$, inverse power-law $F=\alpha \phi^{-n}$, and logarithmic $F=\alpha \ln (\phi)$ functions. We have found wormhole solutions in every single case studied. Due to the qualitative similarity of the obtained behavior for the metric functions and scalar field, in this work we will mainly focus on the presentation of results for the cases with coupling functions $F=\alpha e^{-\phi}$ and $F=\alpha \phi^{2}$ and present combined graphs for different forms of $F(\phi)$ whenever possible. During our quest for regular, physically acceptable wormhole solutions, spontaneously 


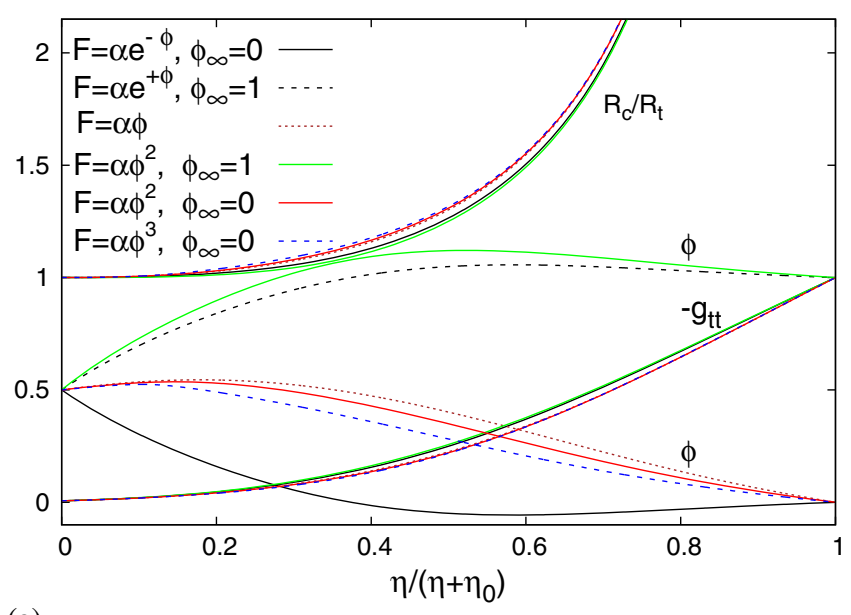

(a)

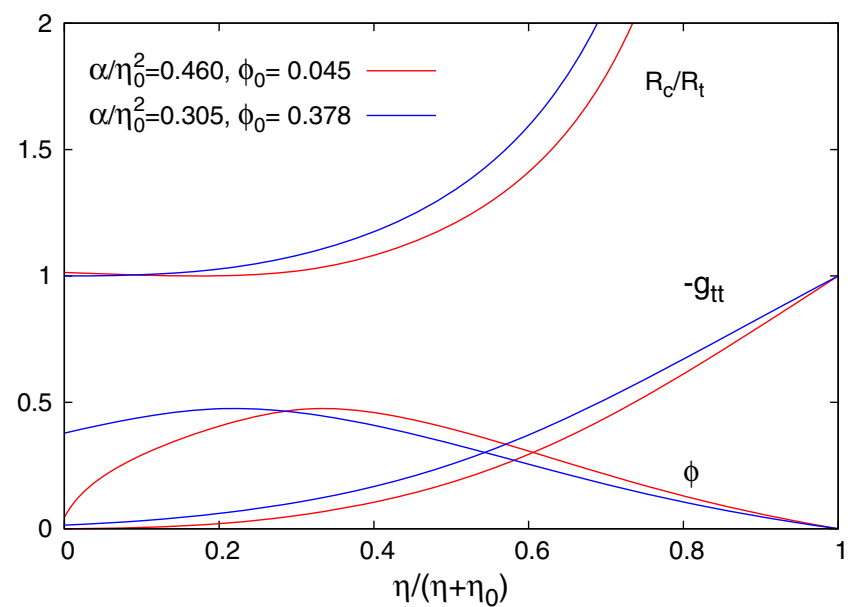

(b)

FIG. 1. Solutions: (a) the metric component $-g_{t t}$, the scalar field $\phi$, and the scaled circumferential radius $R_{c} / R_{t}$ are shown as functions of the compactified coordinate $\eta /(1+\eta)$ for different coupling functions. All solutions are characterized by the same values of $f_{0}(0)$ and $\phi(0)$. (b) The metric component $-g_{t t}$, the scalar field $\phi$, and the scaled circumferential radius $R_{c} / R_{t}$ are shown as function of the compactified coordinate $\eta /(1+\eta)$ for a single-throat wormhole (blue) and a double-throat wormhole (red) for the same values of the scaled scalar charge and the scaled throat area.

scalarized black holes also emerged in multitude, thus confirming the results of [24].

In Fig. 1(a), we depict the metric component $-g_{t t}=e^{f_{0}}$, the scalar field $\phi$, and the scaled circumferential radius $R_{c} / R_{t}$ for several coupling functions $F(\phi)$. All solutions are characterized by the same boundary values $f_{0}(0)=-5$ and $\phi(0)=0.5$, but we have allowed for two different asymptotic values for $\phi$, namely $\phi_{\infty}=0$ and $\phi_{\infty}=1$. We observe that the behavior of the metric component $-g_{t t}$ and the circumferential radius $R_{c} / R_{t}$ depends rather mildly on the form of the coupling function or the asymptotic value $\phi_{\infty}$. On the other hand, both of these factors considerably affect the profile of the scalar field as may be clearly seen from the plot.

We have found both single- and double-throat wormhole solutions for every form of the coupling function $F(\phi)$. In Fig. 1(b), we compare single- and double-throat wormholes for the same values of the scaled scalar charge $D / M$ and scaled throat area $A_{t} / 16 \pi M^{2}$. Once again, it is the scalar field that is mostly affected by the different geometry near the throat or equator. We note for future reference that the derivatives of the $-g_{t t}$ and $\phi$ do not vanish at $\eta=0$, i.e., at the throat, for single-throat wormholes, or at the equator, for double-throat ones. This feature will lead to the introduction of a distribution of matter, albeit a physically acceptable one, at $\eta=0$ when we attempt to symmetrically continue our wormhole solutions to the negative regime of the $\eta$ coordinate. This process and the implications of the associated junction conditions will be studied in Sec. IV.

The spacetime around our wormhole solutions is finite for all values of the radial coordinate $\eta \in[0, \infty)$. All curvature invariant quantities remain everywhere finite, as expected. In Fig. 2(a), we depict the profile of the quantity $F(\phi) R_{G B}^{2}$, for a variety of forms of the coupling function $F(\phi)$ and for the same set of values of the free parameters for easy comparison. We observe that the combination $F(\phi) R_{G B}^{2}$ is indeed finite, vanishes at asymptotic infinity as anticipated while its profile in the small $\eta$ regime depends on the form of $F(\phi)$. We also note that the double-throat solution presents a different profile from the single-throat ones; this is due to the fact that the value of the scalar field at the equator is different from its value at the throat.

Next, we discuss the domain of existence (DOE) of the wormhole solutions, in terms of the scaled scalar charge and the scaled throat area, and restrict our discussion to the indicative cases of the exponential and quadratic coupling functions. In Fig. 2(b), we show the DOE for the exponential case, $F=\alpha e^{-\phi}$. The different curves correspond to families of wormholes for a fixed value of $\alpha$ with single throat (dashed) and double throat (solid). Solutions emerge for arbitrarily small values of $\alpha$ up to some maximal value-here, we depict a variety of solutions arising up to the value $\alpha / \eta_{0}^{2}=0.361$. The boundary of the DOE is formed by the black hole solution with scalar hair (solid black), the wormhole solutions with a degenerate throat (dotted black), configurations with cusp singularities outside the throat (dashed black), and configurations with singularities at the equator (dashed-dotted black). We note that the part of the DOE above the dashed-dotted curve comprises both single- and double-throat wormholes. The single-throat wormholes of this area can in fact be obtained from the double-throat ones-we will return to this point in Sec. IV. The region of the domain of existence below the dashed-dotted curve contains only single-throat wormholes which are not related to double-throat solutions. 


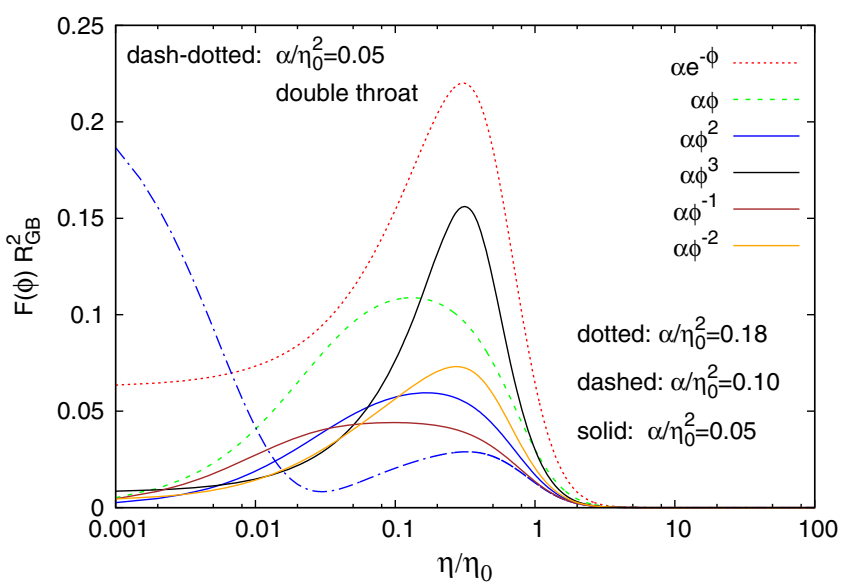

(a)

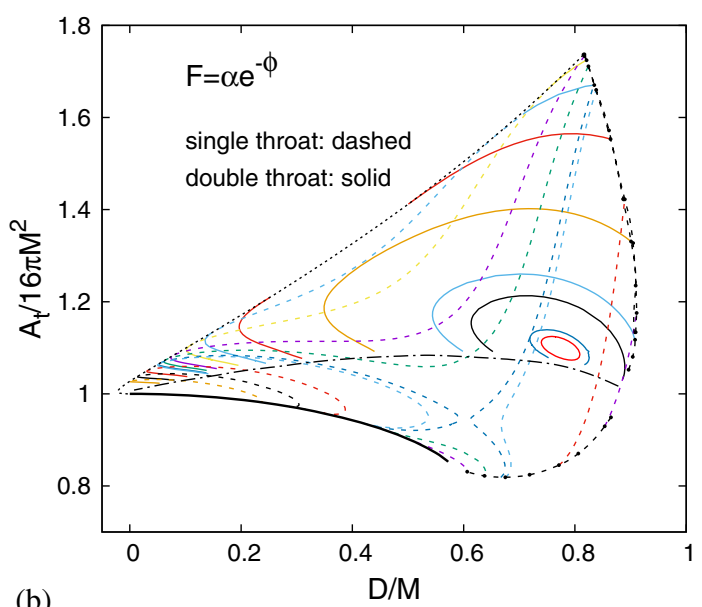

(b)

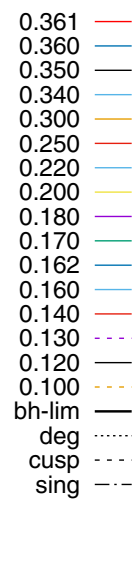

FIG. 2. (a) The quantity $F(\phi) R_{G B}^{2}$ as function of $\eta$ for several forms of the coupling function $F(\phi)$. (b) Domain of existence: the scaled throat area of single- and double-throat wormholes is shown as function of the scaled scalar charge for the coupling function $F=\alpha e^{-\phi}$ for several values of $\alpha / \eta_{0}^{2}$.

We now turn to the case of the quadratic coupling function, $F=\alpha \phi^{2}$. Contrary to what happens in the case of the exponential coupling function, in this case, the DOE depends on the asymptotic value of the scalar field. For $\phi(\infty)=1$, the quantity $\dot{F}(\phi)$ assumes a nonzero asymptotic value, as in the exponential case; therefore the DOE, depicted in Fig. 3(a), is similar to the one displayed in Fig. 2(b). In contrast, if $\phi(\infty)=0$, then $\dot{F}$ vanishes asymptotically and the range of $\alpha$, for which wormholes arise, is also limited from below. The DOE in this case is shown in Fig. 3(b) - now, wormholes emerge only if $0.205<\alpha / \eta_{0}^{2}<0.480$. The Schwarzschild black holes are now part of the boundary of the DOE, as indicated by the dot in Fig. 3(b), since the constant configuration $\phi \equiv \phi_{\infty}=0$ solves the scalar field equation trivially.
Moreover, wormhole solutions exist for which the scalar field may possess $N$ nodes. The boundary of the DOE for $N=1$ is shown in the inlet in Fig. 3(b). Note that the range of $\alpha$ in this case is approximately $1.85 \leq \alpha / \eta_{0}^{2} \leq 2.75$, i.e., considerably larger than for $N=0$.

Let us finally address the issue of the violation of the null and weak energy conditions. In Fig. 4(a), we display the quantity $-T_{t}^{t}+T_{\eta}^{\eta}$ for a number of wormhole solutions arising for different forms of the coupling function $F(\phi)$. It is evident that the NEC is always violated near the throat of each solution by an amount which depends on the form of the coupling function $F(\phi)$ since the latter determines the weight of the GB term in the theory. On the other hand, the NEC is obeyed at asymptotic infinity. We note that, in the case of the double-throat solution, the NEC is violated
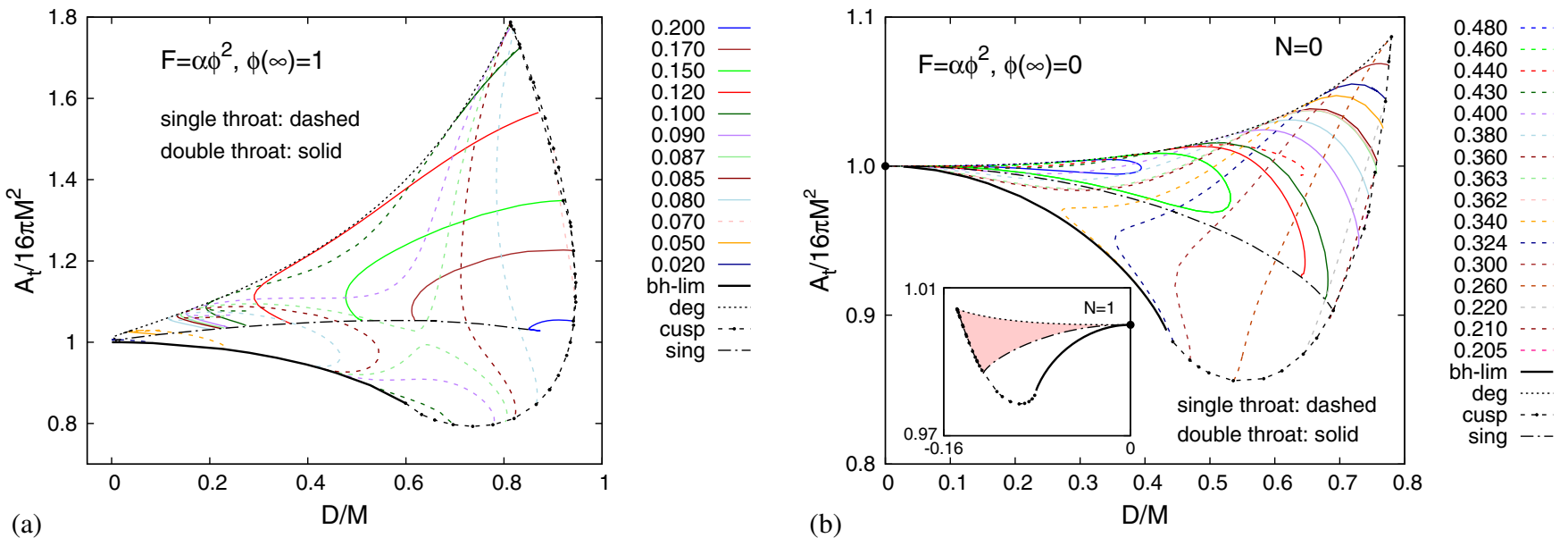

FIG. 3. Domain of existence for the coupling function $F=\alpha \phi^{2}$ for several values of $\alpha / \eta_{0}^{2}$ : the scaled throat area of single- and doublethroat wormholes is shown as function of the scaled scalar charge for (a) $\phi_{\infty}=1$ and (b) $\phi_{\infty}=0$. The dot indicates the Schwarzschild black hole. The inlet shows the domain of existence for wormholes with one node of the scalar field-the red area indicates the domain where single- and double-throat wormholes coexist. 

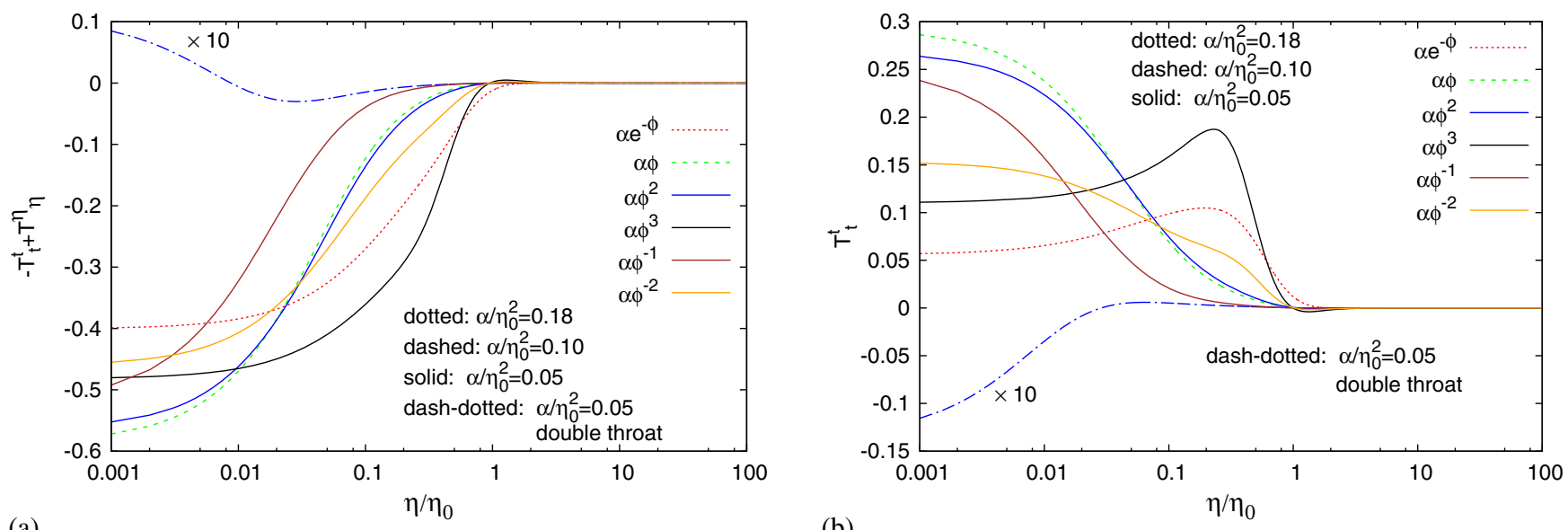

(a)

(b)

FIG. 4. (a) The null energy condition and (b) the weak energy condition for a variety of forms of the coupling function $F(\phi)$.

at the throat while it is obeyed at the equator, according to the analysis of the previous section. A similar behavior is exhibited by the $T_{t}^{t}$ component depicted in Fig. 4(b): the WEC is again violated at the small $\eta$ regime, by an amount determined by $F(\phi)$, while it is obeyed at asymptotic infinity where the GB term becomes negligible. The double-throat solution again respects the WEC at the equator while it violates it near the throat.

\section{JUNCTION CONDITIONS}

Wormhole solutions may be either symmetric or asymmetric under the change $\eta \rightarrow-\eta$. In the context of the EsGB theory with an exponential coupling function $[39,40]$, asymmetric wormholes were found but they were plagued by curvature singularities lurking behind the throat. A regular wormhole solution may then be constructed by imposing a symmetry under the change $\eta \rightarrow-\eta$. The obtained solution then consists of two parts: the first coincides with the part of the asymmetric solution

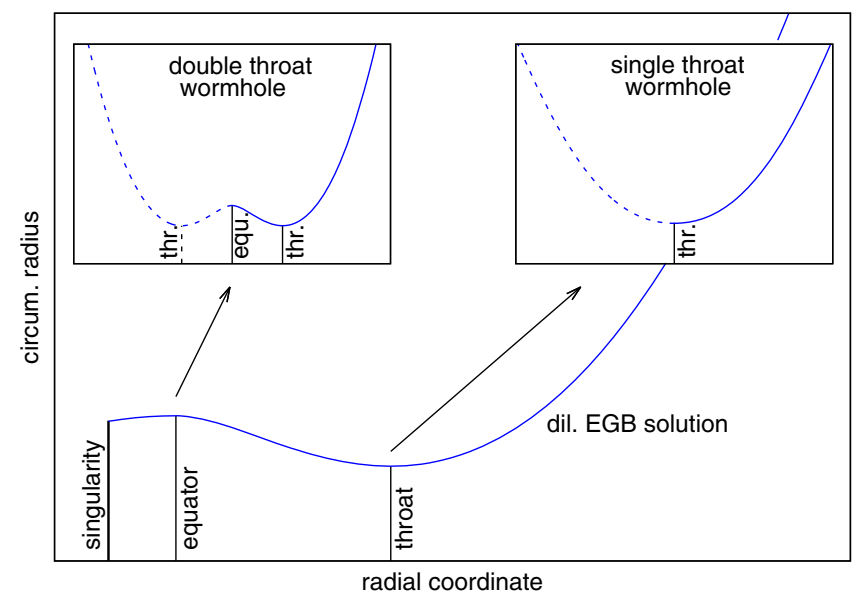

FIG. 5. Schematic picture for the construction of double- and single-throat wormhole solutions. which extends from the asymptotic region at infinity to the location of the throat; the second part of the wormhole solution is obtained by the symmetric continuation of the first part in the negative $\eta$ regime.

A similar construction was performed in the context of the present analysis, in the case of solutions with a single throat - these solutions are the ones depicted in Figs. 2(b), $3(a)$, and 3(b) under the dashed-dotted curves. In the case of singular wormhole solutions with a throat and an equator, a similar process may give rise to double-throat wormholes and to single-throat wormholes since now there are two options, as Fig. 5 depicts. The first option is to construct a regular wormhole by cutting at the throat and symmetrically continuing to the left, as described above; in that case, the equator is removed from the spacetime geometry and a single-throat wormhole is constructed. The second option is to cut the singular solution at the equator, keep the regular part from the asymptotic infinity to the equator and continue symmetrically to the left; in this way, a doublethroat wormhole solution, with an equator located exactly between the throats, is constructed. Both wormholes possess the same mass and scalar charge, since these quantities are extracted from the asymptotic region that is common in both solutions. Hence, for any double-throat wormhole, there exists a single-throat wormhole with the same mass and scalar charge-these are the solutions depicted in Figs. 2(b), 3(a), and 3(b) above the dasheddotted curves.

Let us now discuss in more detail the construction of symmetric, regular, and thus traversable wormholes [49]. From Fig. 1, we observe that the derivatives of the $-g_{t t}$ and $\phi$ do not vanish in general at $\eta=0$. Therefore, imposing a symmetry under $\eta \rightarrow-\eta$ creates a "cusp" in the profile of the aforementioned quantities. This feature may be attributed to the presence of a distribution of matter at $\eta=0$, i.e., around the throat or the equator, for single- or double-throat solutions, respectively. The embedding of this thin-shell matter distribution in the context of the complete solution is 


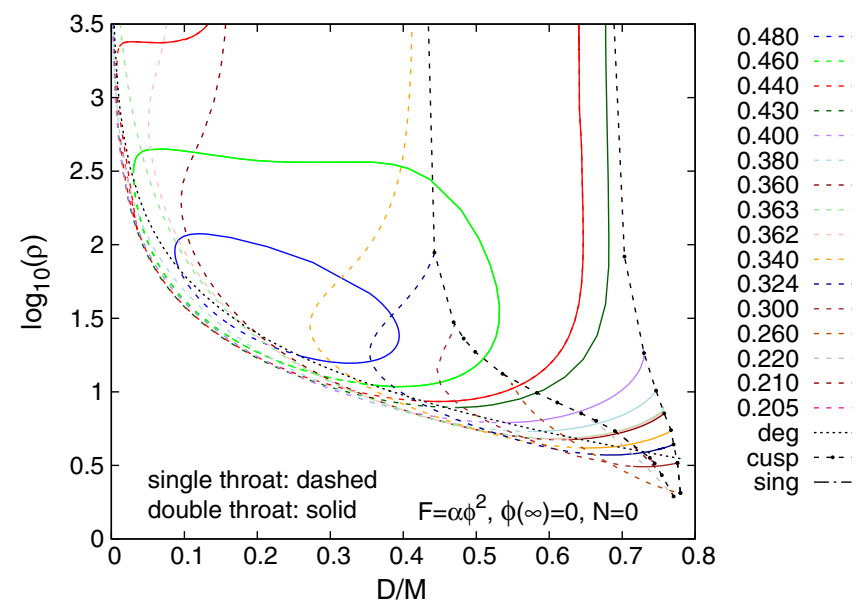

FIG. 6. The energy density $\rho$ at $\eta=0$, for $F(\phi)=\alpha \phi^{2}$ for several values of $\alpha / \eta_{0}^{2}, p=0$ (dust), and specific values of $\left(\lambda_{0}, \lambda_{1}\right)$.

determined through the junction conditions [51,52], that follow by considering the jumps in the Einstein and scalarfield equations (3) as $\eta \rightarrow-\eta$. These are found to have the form

$$
\left\langle G^{\mu}{ }_{\nu}-T^{\mu}{ }_{\nu}\right\rangle=s^{\mu}{ }_{\nu}, \quad\left\langle\nabla^{2} \phi+\dot{F} R_{\mathrm{GB}}^{2}\right\rangle=s_{\text {scal }},
$$

where $s_{\nu}^{\mu}$ denotes the stress-energy tensor of the matter at the throat, respectively, equator, and $s_{\text {scal }}$ a source term for the scalar field. For a physically acceptable solution, this matter distribution should not be exotic. We thus assume a perfect fluid with pressure $p$ and energy density $\rho$, and a scalar charge $\rho_{\text {scal }}$ at the throat, respectively, equator, together with the gravitational source $[39,40]$

$$
S_{\Sigma}=\int\left[\lambda_{1}+2 \lambda_{0} F(\phi) \bar{R}\right] \sqrt{-\bar{h}} d^{3} x
$$

where $\lambda_{1}, \lambda_{0}$ are constants, $\bar{h}_{a b}$ is the three-dimensional induced metric at the throat, respectively, equator, and $\bar{R}$ is the corresponding Ricci scalar. Substitution of the metric then yields the junction conditions

$$
\begin{gathered}
8 \dot{F} \phi^{\prime} e^{-\frac{3 f_{1}}{2}}=\lambda_{1} \eta_{0}^{2}+4 \lambda_{0} F e^{-f_{1}}-\rho \eta_{0}^{2}, \\
e^{-\frac{f_{1}}{2}} f_{0}^{\prime}=\lambda_{1}+p, \\
e^{-f_{1}} \phi^{\prime}-4 \frac{\dot{F}}{\eta_{0}^{2}} f_{0}^{\prime} e^{-2 f_{1}}=-4 \lambda_{0} \frac{\dot{F}}{\eta_{0}^{2}} e^{-\frac{3 f_{1}}{2}}+\frac{\rho_{\text {scal }}}{2},
\end{gathered}
$$

where all quantities are taken at $\eta=0$. The above junction conditions determine $\rho, p$, and $\rho_{\text {scal }}$ in terms of the arbitrary constants $\lambda_{0}$ and $\lambda_{1}$ and the form of the scalar field and metric functions close to the boundary.
For every form of the coupling function $F(\phi)$, we may find an extensive $\left(\lambda_{0}, \lambda_{1}\right)$-parameter regime over which $\rho$ is always positive, and the necessity of the exotic matter is thus avoided. An interesting special case is when the matter distribution around the throat/equator has a vanishing pressure, i.e., $p=0$, and therefore its equation of state is the one of dust. In this case, Eq. (41) gives $\lambda_{1}=e^{-f_{1} / 2} f_{0}^{\prime}$. If we choose $\lambda_{0}=\lambda_{1}$, Eqs. (40) and (42) easily yield

$$
\rho=\frac{e^{-\frac{3 f_{1}}{2}}}{\eta_{0}}\left[\left(4 F+\eta_{0}^{2} e^{f_{1}}\right) f_{0}^{\prime}-8 \dot{F} \phi^{\prime}\right], \quad \rho_{\mathrm{sc}}=2 e^{-f_{1}} \phi^{\prime},
$$

respectively, where again all quantities are evaluated at $\eta=0$. In Fig. 6, we depict the energy density $\rho$ at the throat, respectively, equator, as a function of the scaled scalar charge $D / M$, for a variety of wormhole solutions arising for $F(\phi)=\alpha \phi^{2}$ and for the aforementioned values of $p, \lambda_{0}$ and $\lambda_{1}$. We note that in this example the energy density $\rho$ is positive for all wormhole solutions. As in the construction of the solution, where the synergy of an ordinary distribution of matter with a gravitational source kept the throat, respectively, equator, open, here a similar synergy creates a symmetric wormhole free of singularities.

\section{EMBEDDING DIAGRAM}

A useful way to visualize the geometry of a given manifold is the construction of the corresponding embedding diagram. In this case, we consider the isometric embedding of the equatorial plane of our wormhole solutions, defined as the line element (5) for $\mathrm{t}=$ const. and $\theta=\pi / 2$. The isometric embedding follows by equating the line element of the two-dimensional equatorial plane with a hypersurface in the three-dimensional Euclidean space, namely,

$$
e^{f_{1}}\left[d \eta^{2}+\left(\eta^{2}+\eta_{0}^{2}\right) d \varphi^{2}\right]=d z^{2}+d w^{2}+w^{2} d \varphi^{2},
$$

where $(z, w, \varphi)$ is a set of cylindrical coordinates on the hypersurface. Considering $z$ and $w$ as functions of $\eta$, we find

$$
\begin{gathered}
w=e^{f_{1} / 2} \sqrt{\eta^{2}+\eta_{0}^{2}} \\
\left(\frac{d w}{d \eta}\right)^{2}+\left(\frac{d z}{d \eta}\right)^{2}=e^{f_{1}}
\end{gathered}
$$

Then, combining the above equations, we find

$$
z(\eta)= \pm \int_{0}^{\eta} \sqrt{e^{f_{1}(\tilde{\eta})}-\left(\frac{d}{d \tilde{\eta}}\left[e^{f_{1}(\tilde{\eta}) / 2} \sqrt{\tilde{\eta}^{2}+\eta_{0}^{2}}\right]\right)^{2}} d \tilde{\eta}
$$




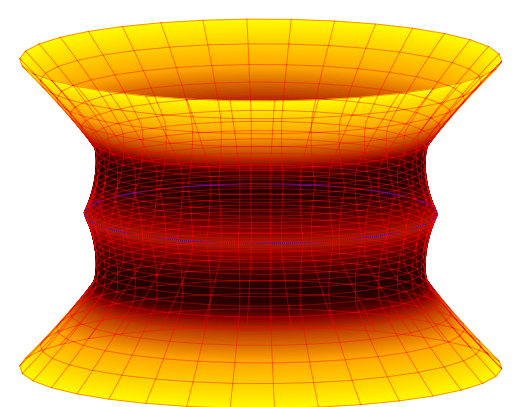

(a)

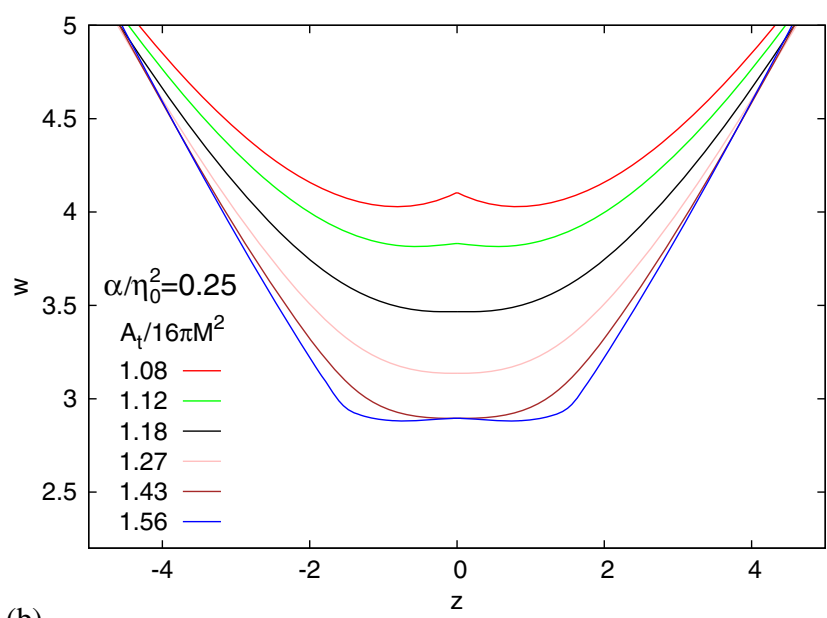

(b)

FIG. 7. (a) The embedded equatorial plane is shown for the double-throat wormhole with $\alpha / \eta_{0}^{2}=0.35$ and $D / M=0.886$. (b) The profiles of the isometric embedding are shown for a sequence of solutions for the coupling function $F=0.25 e^{-\phi}$.

Therefore, $\{w(\eta), z(\eta)\}$ is a parametric representation of a slice of the embedded $\theta=\pi / 2$ plane for a fixed value of the $\varphi$ coordinate, while the corresponding surface of revolution is the three-dimensional representation of the wormhole's geometry.

In Fig. 7(a), we depict the isometric embedding of the geometry of a symmetric, traversable, double-throat wormhole solution. The three-dimensional view of the surface follows from the parametric plot $(w(\eta) \cos \varphi$, $w(\eta) \sin \varphi, z(\eta))$ as described above. The diagram clearly features an equator and two throats smoothly connected to two asymptotic regimes. In Fig. 7(b), we also show the geometry transition between single- and double-throat wormholes, by plotting $w$ versus $z$, for a sequence of solutions for fixed $\alpha / \eta_{0}^{2}=0.25$. We observe that, with increasing scaled throat area, the double-throat wormholes develop a degenerate throat and turn into single-throat ones. If the scaled throat area is increased further, a second transition takes place where the single-throat wormholes turn again to double-throat ones.

\section{CONCLUSIONS AND DISCUSSION}

In this work, we have considered a general class of EsGB theories with an arbitrary coupling function between the scalar field and the quadratic Gauss-Bonnet term. By employing a novel coordinate system, we have allowed for wormhole solutions with either single- or double-throat geometries to emerge. We have determined the asymptotic form of the metric functions and scalar field in the small and large radial-coordinate regimes, and demonstrated that the null and weak energy conditions may be violated, especially in the inner regime where the effect of the GB term is dominant.

We have then numerically integrated our set of field equations in order to determine the complete wormhole solutions that interpolate between the derived asymptotic solutions. We have found wormholes, with either a single throat or a double throat and an equator, for every form of the coupling function we have tried. The spacetime is regular over the entire positive range of the radial coordinate, as also is the nontrivial scalar field that characterizes every wormhole solution. Our solutions are therefore characterized by two independent parameters, their mass and scalar charge. The domain of existence has been studied in detail in each case, and here we have presented the ones for the exponential and quadratic coupling functions in order to discuss the qualitative differences as the form of the coupling function and the value of the scalar field at asymptotic infinity varies.

An important result of our analysis is that the EsGB theories always feature wormhole solutions without the need for exotic matter, since the higher-curvature terms allow for gravitational effective negative energy densities. This has been demonstrated by examining the null and weak energy conditions for our solutions and showing that indeed the coupling between the scalar field and the GB term results in a negative energy density near the throat/ equator. The null energy condition is also violated since it is associated with the appearance of a throat that every wormhole solution must possess.

In order to construct traversable wormhole solutions with no spacetime singularities beyond the throat or equator, our regular solution over the positive range of the radial coordinate was extended in the negative range in a symmetric way. This construction demands the introduction of a distribution of matter around the throat or equator that nevertheless may be shown to consist of physically acceptable particles. We have provided an indicative example where this distribution of matter is described by the equation of state of dust with a vanishing isotropic pressure and a positive energy density. 
Let us address at this point the issue of the existing bounds on the GB coupling constant. The parameters of any modified gravitational theory, including the EsGB theory, may be constrained by processes and observations in strong gravitational regimes. The most recent bound on the GB coupling parameter $\alpha$ was set in [53] where the effect of the scalar dipole radiation on the phase evolution of the gravitational waveform was taken into account- - this radiation was emitted during the merging process of two binary systems in which one of the constituents is a scalarized black hole (GW151226 and GW170608 as detected by LIGO). This bound was set on the value $\sqrt{\alpha}<10.1 \mathrm{~km}$, taking into account the different definitions of $\alpha$; in dimensionless units, this translates to $\alpha / M^{2}<1.72$, where $M$ is the characteristic mass scale of the system, i.e., the black-hole mass. In the absence of a direct bound on wormholes, since no such object has been detected so far, and demanding that the EsGB theory should allow for both black-hole solutions and wormholes to emerge, we apply the aforementioned bound by LIGO on our wormhole solutions too. For an exponential coupling function, all of our solutions satisfy the bound $\alpha / M^{2}<0.91$, while for a quadratic coupling function we obtain $\alpha / M^{2}<0.605$ (for solutions with no nodes for the scalar field), respectively, $\alpha / M^{2}<2.9$ (for solutions with one node). Thus the observational bound leaves unaffected the aforementioned DOEs: all solutions in Figs. 2(b), 3(a), and 3(b) (with no nodes) fall entirely within the allowed range.
Our next step will be to study the physical characteristics of our solutions in greater detail and to generalize them to admit also rotation [47]. In addition, a linear stability analysis of these EsGB wormholes [39,40,54,55] will be performed and their radial and quasinormal modes, which could be observable signatures of their existence, will be determined.

\section{ACKNOWLEDGMENTS}

G. A. would like to thank Onassis Foundation for the financial support provided through its scholarship program. This research is cofinanced by Greece and the European Union (European Social Fund) through the Operational Programme "Human Resources Development, Education and Lifelong Learning" in the context of the project "Strengthening Human Resources Research Potential via Doctorate Research" (Grant No. MIS-5000432) implemented by the State Scholarships Foundation (IKY). B. K. and J.K. gratefully acknowledge support by the DFG Research Training Group 1620 Models of Gravity and the COST Action CA16104. B. K. acknowledges helpful discussions with Eugen Radu.

\section{APPENDIX: FIELD EQUATIONS}

Employing the metric (5) in Eq. (3), the $(t t),(\eta \eta)$, and $(\theta \theta)$ components of Einstein's equations take the form

$$
\begin{aligned}
& \eta_{0}^{6}\left(2 f_{1}^{\prime}\left(\phi^{\prime}\left(\dot{F}\left(f_{1}^{\prime 2}-4 f_{1}^{\prime \prime}\right)-2 f_{1}^{\prime} \ddot{F} \phi^{\prime}\right)-2 \dot{F} f_{1}^{\prime} \phi^{\prime \prime}\right)+e^{f_{1}}\left(f_{1}^{\prime 2}+4 f_{1}^{\prime \prime}+\phi^{\prime 2}\right)\right)+\eta^{4}\left[-4 \dot{F} \eta f_{1}^{\prime} \phi^{\prime \prime}\left(\eta f_{1}^{\prime}+4\right)\right. \\
& \left.\quad+e^{f_{1}} \eta\left(\eta\left(4 f_{1}^{\prime \prime}+\phi^{\prime 2}\right)+\eta f_{1}^{\prime 2}+8 f_{1}^{\prime}\right)+2 \phi^{\prime}\left(\dot{F}\left(f_{1}^{\prime}\left(\eta^{2} f_{1}^{\prime 2}-8\right)-4 \eta f_{1}^{\prime \prime}\left(\eta f_{1}^{\prime}+2\right)\right)-2 \eta f_{1}^{\prime} \ddot{F} \phi^{\prime}\left(\eta f_{1}^{\prime}+4\right)\right)\right] \\
& \quad+\eta_{0}^{4}\left[2 \phi^{\prime}\left(\dot{F}\left(3 f_{1}^{\prime}\left(\eta^{2} f_{1}^{\prime 2}-4\right)-4 \eta f_{1}^{\prime \prime}\left(3 \eta f_{1}^{\prime}+2\right)\right)-2 \ddot{F} \phi^{\prime}\left(\eta f_{1}^{\prime}+2\right)\left(3 \eta f_{1}^{\prime}-2\right)\right)-4 \dot{F} \phi^{\prime \prime}\left(\eta f_{1}^{\prime}+2\right)\left(3 \eta f_{1}^{\prime}-2\right)\right. \\
& \left.\quad+e^{f_{1}}\left(\eta\left(3 \eta\left(4 f_{1}^{\prime \prime}+\phi^{\prime 2}\right)+3 \eta f_{1}^{\prime 2}+8 f_{1}^{\prime}\right)+4\right)\right]+\eta \eta_{0}^{2}\left[-4 \eta \ddot{F} \phi^{\prime 2}\left(\eta f_{1}^{\prime}\left(3 \eta f_{1}^{\prime}+8\right)-4\right)+e^{f_{1}} \eta\left(4+\eta\left(16 f_{1}^{\prime}\right.\right.\right. \\
& \left.\left.\quad+3 \eta f_{1}^{\prime 2}+3 \eta\left(\phi^{\prime 2}+4 f_{1}^{\prime \prime}\right)\right)\right)+2 \dot{F}\left(\phi^{\prime}\left(3 \eta^{3}\left(f_{1}^{\prime}\right)^{3}-4 \eta^{2} f_{1}^{\prime \prime}\left(3 \eta f_{1}^{\prime}+4\right)-20 \eta f_{1}^{\prime}-16\right)\right. \\
& \left.\quad-2 \eta \phi^{\prime \prime}\left(\eta f_{1}^{\prime}\left(3 \eta f_{1}^{\prime}+8\right)-4\right)\right)=0 \\
& \quad e^{f_{1}}\left[\eta^{3}\left(2 f_{0}^{\prime}\left(\eta f_{1}^{\prime}+2\right)+f_{1}^{\prime}\left(\eta f_{1}^{\prime}+4\right)-\eta \phi^{\prime 2}\right)+2 \eta_{0}^{2}\left(\eta\left(2 f_{0}^{\prime}\left(\eta f_{1}^{\prime}+1\right)+f_{1}^{\prime}\left(\eta f_{1}^{\prime}+2\right)-\eta \phi^{\prime 2}\right)-2\right)\right. \\
& \left.\quad+\eta_{0}^{4}\left(f_{1}^{\prime 2}+2 f_{0}^{\prime} f_{1}^{\prime}-\phi^{\prime 2}\right)\right]-2 \dot{F} f_{0}^{\prime} \phi^{\prime}\left(12\left(\eta^{2}+\eta_{0}^{2}\right) \eta f_{1}^{\prime}+3\left(\eta^{2}+\eta_{0}^{2}\right)^{2} f_{1}^{\prime 2}+8 \eta^{2}-4 \eta_{0}^{2}\right)=0, \\
& \quad \eta^{3}\left[-4 \dot{F} f_{0}^{\prime} \phi^{\prime \prime}\left(\eta f_{1}^{\prime}+2\right)-4 f_{0}^{\prime} \ddot{F} \phi^{\prime 2}\left(\eta f_{1}^{\prime}+2\right)-2 \dot{F} \phi^{\prime}\left(-2 f_{0}^{\prime}\left(\eta f_{1}^{\prime 2}-\eta f_{1}^{\prime \prime}+f_{1}^{\prime}\right)+2 f_{0}^{\prime \prime}\left(\eta f_{1}^{\prime}+2\right)+f_{0}^{\prime 2}\left(\eta f_{1}^{\prime}+2\right)\right)\right. \\
& \left.+e^{f_{1}}\left(\eta\left(2\left(f_{0}^{\prime \prime}+f_{1}^{\prime \prime}\right)+\phi^{\prime 2}\right)+\eta f_{0}^{\prime 2}+2 f_{0}^{\prime}+2 f_{1}^{\prime}\right)\right]+2 \eta_{0}^{2}\left[e^{f_{1}}\left(\eta\left(\eta\left(2\left(f_{0}^{\prime \prime}+f_{1}^{\prime \prime}\right)+\phi^{\prime 2}\right)+\eta f_{0}^{\prime 2}+f_{0}^{\prime}+f_{1}^{\prime}\right)+2\right)\right. \\
& \left.-2 \dot{F} \phi^{\prime}\left(f_{0}^{\prime}\left(2-\eta\left(2 \eta f_{1}^{\prime 2}-2 \eta f_{1}^{\prime \prime}+f_{1}^{\prime}\right)\right)+2 \eta f_{0}^{\prime \prime}\left(\eta f_{1}^{\prime}+1\right)+\eta f_{0}^{\prime 2}\left(\eta f_{1}^{\prime}+1\right)\right)-4 \eta f_{0}^{\prime}\left(\eta f_{1}^{\prime}+1\right)\left(\dot{F} \phi^{\prime \prime}+\ddot{F} \phi^{\prime 2}\right)\right] \\
& +\eta_{0}^{4}\left[-4 f_{0}^{\prime} f_{1}^{\prime} \ddot{F} \phi^{\prime 2}-4 \dot{F} f_{0}^{\prime} f_{1}^{\prime} \phi^{\prime \prime}-2 \dot{F} \phi^{\prime}\left(f_{1}^{\prime}\left(f_{0}^{\prime 2}-2 f_{1}^{\prime} f_{0}^{\prime}+2 f_{0}^{\prime \prime}\right)+2 f_{0}^{\prime} f_{1}^{\prime \prime}\right)+e^{f_{1}}\left(f_{0}^{\prime 2}+2\left(f_{0}^{\prime \prime}+f_{1}^{\prime \prime}\right)+\phi^{\prime 2}\right)\right]=0,
\end{aligned}
$$

respectively. The scalar equation in turn yields 


$$
\begin{aligned}
\eta_{0}^{6} & \left(f_{0}^{\prime}\left(4 \dot{F} f_{1}^{\prime} f_{1}^{\prime \prime}-\dot{F} f_{1}^{\prime 3}+e^{f_{1}} \phi^{\prime}\right)+2 \dot{F} f_{1}^{\prime 2} f_{0}^{\prime \prime}+\dot{F} f_{0}^{\prime 2} f_{1}^{\prime 2}+2 e^{f_{1}} \phi^{\prime \prime}+e^{f_{1}} f_{1}^{\prime} \phi^{\prime}\right)+\eta^{4}\left[\dot{F} \eta f_{0}^{\prime 2} f_{1}^{\prime}\left(\eta f_{1}^{\prime}+4\right)\right. \\
& +f_{0}^{\prime}\left(4 \dot{F} f_{1}^{\prime}\left(\eta^{2} f_{1}^{\prime \prime}+2\right)+\eta\left(\dot{F}\left(8 f_{1}^{\prime \prime}-\eta f_{1}^{\prime 3}\right)+e^{f_{1}} \eta \phi^{\prime}\right)\right)+\eta\left(2\left(\dot{F}\left(\eta f_{1}^{\prime 2} f_{0}^{\prime \prime}+4 f_{1}^{\prime} f_{0}^{\prime \prime}\right)+e^{f_{1}} \eta \phi^{\prime \prime}\right)\right. \\
& \left.+e^{f_{1}} \phi^{\prime}\left(\eta f_{1}^{\prime}+4\right)\right]+\eta_{0}^{4}\left[f_{0}^{\prime}\left(12 \dot{F} f_{1}^{\prime}\left(\eta^{2} f_{1}^{\prime \prime}+1\right)-3 \dot{F} \eta^{2} f_{1}^{\prime 3}+\eta\left(8 \dot{F} f_{1}^{\prime \prime}+3 e^{f_{1}} \eta \phi^{\prime}\right)\right)+6 \dot{F} \eta^{2} f_{1}^{\prime 2} f_{0}^{\prime \prime}+8 \dot{F} \eta f_{1}^{\prime} f_{0}^{\prime \prime}\right. \\
& \left.-8 \dot{F} f_{0}^{\prime \prime}+6 e^{f_{1}} \eta^{2} \phi^{\prime \prime}+\dot{F} f_{0}^{\prime 2}\left(3 \eta^{2} f_{1}^{\prime 2}+4 \eta f_{1}^{\prime}-4\right)+e^{f_{1}} \eta \phi^{\prime}\left(3 \eta f_{1}^{\prime}+4\right)\right]+\eta \eta_{0}^{2}\left[\dot{F} \eta f_{0}^{\prime 2}\left(3 \eta^{2} f_{1}^{\prime 2}+8 \eta f_{1}^{\prime}-4\right)\right. \\
& +f_{0}^{\prime}\left(\dot{F}\left(-3 \eta^{3} f_{1}^{\prime 3}+4 \eta f_{1}^{\prime}\left(3 \eta^{2} f_{1}^{\prime \prime}+5\right)+16\left(\eta^{2} f_{1}^{\prime \prime}+1\right)\right)+3 e^{f_{1}} \eta^{3} \phi^{\prime}\right)+\eta\left(2 \dot{F} f_{0}^{\prime \prime}\left(3 \eta^{2} f_{1}^{\prime 2}+8 \eta f_{1}^{\prime}-4\right)\right. \\
& \left.\left.+6 e^{f_{1}} \eta^{2} \phi^{\prime \prime}+e^{f_{1}} \eta \phi^{\prime}\left(3 \eta f_{1}^{\prime}+8\right)\right)\right]=0 .
\end{aligned}
$$

In the above equations, the prime denotes differentiation with respect to the radial coordinate $\eta$.

[1] E. Berti et al., Classical Quantum Gravity 32, 243001 (2015).

[2] T. P. Sotiriou, Lect. Notes Phys. 892, 3 (2015).

[3] G. W. Horndeski, Int. J. Theor. Phys. 10, 363 (1974).

[4] C. Charmousis, E. J. Copeland, A. Padilla, and P. M. Saffin, Phys. Rev. Lett. 108, 051101 (2012).

[5] T. Kobayashi, M. Yamaguchi, and J. Yokoyama, Prog. Theor. Phys. 126, 511 (2011).

[6] J. Sakstein and B. Jain, Phys. Rev. Lett. 119, 251303 (2017).

[7] B. Zwiebach, Phys. Lett. B 156, 315 (1985).

[8] D. J. Gross and J. H. Sloan, Nucl. Phys. B291, 41 (1987).

[9] R. R. Metsaev and A. A. Tseytlin, Nucl. Phys. B293, 385 (1987).

[10] P. Kanti, N. E. Mavromatos, J. Rizos, K. Tamvakis, and E. Winstanley, Phys. Rev. D 54, 5049 (1996).

[11] T. Torii, H. Yajima, and K. I. Maeda, Phys. Rev. D 55, 739 (1997).

[12] Z. K. Guo, N. Ohta, and T. Torii, Prog. Theor. Phys. 120, 581 (2008).

[13] P. Pani and V. Cardoso, Phys. Rev. D 79, 084031 (2009).

[14] P. Pani, C. F. B. Macedo, L. C. B. Crispino, and V. Cardoso, Phys. Rev. D 84, 087501 (2011).

[15] B. Kleihaus, J. Kunz, and E. Radu, Phys. Rev. Lett. 106, 151104 (2011).

[16] D. Ayzenberg, K. Yagi, and N. Yunes, Phys. Rev. D 89, 044023 (2014).

[17] D. Ayzenberg and N. Yunes, Phys. Rev. D 90, 044066 (2014).

[18] A. Maselli, P. Pani, L. Gualtieri, and V. Ferrari, Phys. Rev. D 92, 083014 (2015).

[19] B. Kleihaus, J. Kunz, and S. Mojica, Phys. Rev. D 90, 061501 (2014).

[20] B. Kleihaus, J. Kunz, S. Mojica, and E. Radu, Phys. Rev. D 93, 044047 (2016).

[21] J. L. Blázquez-Salcedo, C. F. B. Macedo, V. Cardoso, V. Ferrari, L. Gualtieri, F. S. Khoo, J. Kunz, and P. Pani, Phys. Rev. D 94, 104024 (2016).

[22] T. P. Sotiriou and S. Y. Zhou, Phys. Rev. Lett. 112, 251102 (2014).

[23] T. P. Sotiriou and S. Y. Zhou, Phys. Rev. D 90, 124063 (2014).
[24] G. Antoniou, A. Bakopoulos, and P. Kanti, Phys. Rev. Lett. 120, 131102 (2018); Phys. Rev. D 97, 084037 (2018).

[25] D. D. Doneva and S. S. Yazadjiev, Phys. Rev. Lett. 120, 131103 (2018).

[26] H. O. Silva, J. Sakstein, L. Gualtieri, T. P. Sotiriou, and E. Berti, Phys. Rev. Lett. 120, 131104 (2018).

[27] G. Antoniou, A. Bakopoulos, and P. Kanti, Phys. Rev. D 97, 084037 (2018).

[28] H. Witek, L. Gualtieri, P. Pani, and T. P. Sotiriou, Phys. Rev. D 99, 064035 (2019).

[29] M. Minamitsuji and T. Ikeda, Phys. Rev. D 99, 044017 (2019).

[30] H. O. Silva, C. F. B. Macedo, T. P. Sotiriou, L. Gualtieri, J. Sakstein, and E. Berti, Phys. Rev. D 99, 064011 (2019).

[31] A. Bakopoulos, G. Antoniou, and P. Kanti, Phys. Rev. D 99, 064003 (2019).

[32] Y. Brihaye and L. Ducobu, Phys. Lett. B 795, 135 (2019).

[33] C. F. B. Macedo, J. Sakstein, E. Berti, L. Gualtieri, H. O. Silva, and T.P. Sotiriou, Phys. Rev. D 99, 104041 (2019).

[34] D. D. Doneva, K. V. Staykov, and S. S. Yazadjiev, Phys. Rev. D 99, 104045 (2019).

[35] Y. S. Myung and D. C. Zou, Int. J. Mod. Phys. D 28, 1950114 (2019).

[36] P. V. P. Cunha, C. A. R. Herdeiro, and E. Radu, Phys. Rev. Lett. 123, 011101 (2019).

[37] Y. Brihaye, B. Hartmann, N. P. Aprile, and J. Urrestilla, arXiv:1911.01950.

[38] J. L. Blázquez-Salcedo, D. D. Doneva, J. Kunz, and S. S. Yazadjiev, Phys. Rev. D 98, 084011 (2018).

[39] P. Kanti, B. Kleihaus, and J. Kunz, Phys. Rev. Lett. 107, 271101 (2011).

[40] P. Kanti, B. Kleihaus, and J. Kunz, Phys. Rev. D 85, 044007 (2012).

[41] M. S. Morris and K. S. Thorne, Am. J. Phys. 56, 395 (1988).

[42] M. Visser, Lorentzian Wormholes: From Einstein to Hawking (AIP, Woodbury, USA, 1995).

[43] H. G. Ellis, J. Math. Phys. (N.Y.) 14, 104 (1973).

[44] H. G. Ellis, Gen. Relativ. Gravit. 10, 105 (1979).

[45] K. A. Bronnikov, Acta Phys. Pol. B 4, 251 (1973).

[46] T. Kodama, Phys. Rev. D 18, 3529 (1978). 
[47] B. Kleihaus and J. Kunz, Phys. Rev. D 90, 121503 (2014).

[48] X. Y. Chew, B. Kleihaus, and J. Kunz, Phys. Rev. D 94, 104031 (2016).

[49] As the coupling function may acquire different forms in the context of our analysis, constructing also asymmetric wormholes, see, e.g., [50], may be indeed a possibility that we plan to pursue in a future work.

[50] K. A. Bronnikov, K. A. Baleevskikh, and M. V. Skvortsova, Phys. Rev. D 96, 124039 (2017).
[51] W. Israel, Nuovo Cimento B 44S10, 1 (1966); 44, 1 (1966); 48, 463 (1967).

[52] S. C. Davis, Phys. Rev. D 67, 024030 (2003).

[53] R. Nair, S. Perkins, H. O. Silva, and N. Yunes, Phys. Rev. Lett. 123, 191101 (2019).

[54] O. A. Evseev and O. I. Melichev, Phys. Rev. D 97, 124040 (2018).

[55] M. A. Cuyubamba, R. A. Konoplya, and A. Zhidenko, Phys. Rev. D 98, 044040 (2018). 